\title{
Minimization of non-common path aberrations at the Palomar telescope using a self-coherent camera
}

\author{
R. Galicher ${ }^{1}$, P. Baudoz ${ }^{1}$, J.-R. Delorme ${ }^{2}$, D. Mawet $^{2}$, M. Bottom ${ }^{3}$, J. K. Wallace ${ }^{3}$, E. Serabyn ${ }^{3}$, and C. Shelton ${ }^{3}$ \\ ${ }^{1}$ Lesia, Observatoire de Paris, PSL Research University, CNRS, Sorbonne Universités, Univ. Paris Diderot, UPMC Univ. Paris 06, \\ Sorbonne Paris Cité, 5 place Jules Janssen, 92190 Meudon, France \\ e-mail: raphael.galicher@obspm.fr \\ 2 Department of Astronomy, California Institute of Technology, Pasadena, CA 91125, USA \\ 3 Jet Propulsion Laboratory, California Institute of Technology, Pasadena, CA 91109, USA
}

Received 10 July 2019 / Accepted 16 September 2019

\begin{abstract}
Context. The two main advantages of exoplanet imaging are the discovery of objects in the outer part of stellar systems, which constrains the models of planet formation, and its ability to spectrally characterize the planets to study their atmospheres. It is, however, challenging because exoplanets are up to $10^{10}$ times fainter than their stars and are separated by a fraction of an arcsecond. Current instruments like SPHERE-VLT or GPI-Gemini detect young and massive planets only because of non-common path aberrations (NCPA) that are not corrected by the adaptive optics system. To probe fainter exoplanets a new instruments capable of minimizing the NCPA is needed. One solution is the self-coherent camera (SCC) focal plane wavefront sensor which is able to attenuate the starlight by factors of up to several $10^{8}$ in the laboratory in space-like conditions.

Aims. In this paper, we demonstrate the SCC on the sky for the first time.

Methods. We installed an SCC on the stellar double coronagraph instrument at the Hale telescope. We minimize the NCPA that limited the vortex coronagraph performance. We then compared this procedure to the standard procedure used at Palomar.

Results. On internal sources, we demonstrated that the SCC improves the coronagraphic detection limit by a factor of 4-20 between 1.5 and $5 \lambda / D$. Using this SCC calibration, the on-sky contrast is improved by a factor of 5 between 2 and $4 \lambda / D$. These results prove the ability of the SCC to be implemented in an existing instrument.

Conclusions. This paper highlights two interests of the self-coherent camera. First, the SCC can minimize the speckle intensity in the field of view, especially the ones that are very close to the star where many exoplanets are to be discovered. Then the SCC has a $100 \%$ efficiency with science time as each image can be used for both science and NCPA minimization.
\end{abstract}

Key words. instrumentation: adaptive optics - instrumentation: high angular resolution - techniques: high angular resolution

\section{Introduction}

Imaging of exoplanets is one priority for astronomers because it is the only technique that can discover long orbital period planets and that enables the spectral characterization of their atmospheres. It is very challenging as the planets are $10^{4}$ to $10^{10} \mathrm{dim}-$ mer than their host stars and at a fraction of arcsecond from them. Many coronagraphs have been proposed to reduce the star diffraction pattern without changing the exoplanet image (Snik et al. 2018). Several are installed on the $8 \mathrm{~m}$ class telescopes in instruments like Spectro Polarimetric High contrast Exoplanet REsearch (SPHERE, Beuzit et al. 2019) and Gemini Planet Imager (GPI, Macintosh et al. 2014) that were built to discover exoplanets by imaging. The coronagraphic images produced by these instruments enable the detection of planets that are up to $\sim 10^{6}$ times fainter than the host star. This performance is far from the best performance reached in the laboratory with attenuation of the starlight by a factor of $10^{9}$ to $10^{10}$ (Baudoz et al. 2018a; Lawson et al. 2013) because wavefront aberrations upstream of the coronagraph can be measured and minimized down to a few picometers rms in the laboratory using the technique of focal plane wavefront sensing and control, for example the pair-wise technique (Give'on et al. 2011) coupled with electric field conjugation (Give'on et al. 2007), speckle nulling (Bordé \& Traub 2006), or the self-coherent camera (Galicher et al. 2008). Behind a ground-based telescope, it is more complicated to control the aberrations because they are not static but quasi-static with respect to the exposure times used to record the coronagraphic image. As a consequence, even using focal plane wavefront control like speckle nulling (Martinache et al. 2014; Bottom et al. 2016a) or electric field conjugation (EFC, Cady et al. 2013; Matthews et al. 2017), the level of aberrations is about $10 \mathrm{~nm}$ rms limiting the starlight attenuation to factor of $\sim 10^{5}$.

In this paper, we present the coronagraphic performance obtained at the Palomar telescope using a self-coherent camera. In Sect. 2 we recall the principle of the self-coherent camera. We then explain how it was implemented in the stellar double coronagraph instrument (Mawet et al. 2014; Bottom et al. 2016b) in Sect. 3. Finally, after presenting the procedures for non-common path aberration (NCPA) calibration in Sect. 4, we present the performance of the self-coherent camera on internal source and on-sky in Sects. 5 and 6.

\section{Principle of the self-coherent camera}

The performance of coronagraphs is limited by phase and amplitude aberrations of the wavefront upstream of the focal plane mask. In ground-based telescopes, the adaptive optics (AO) 


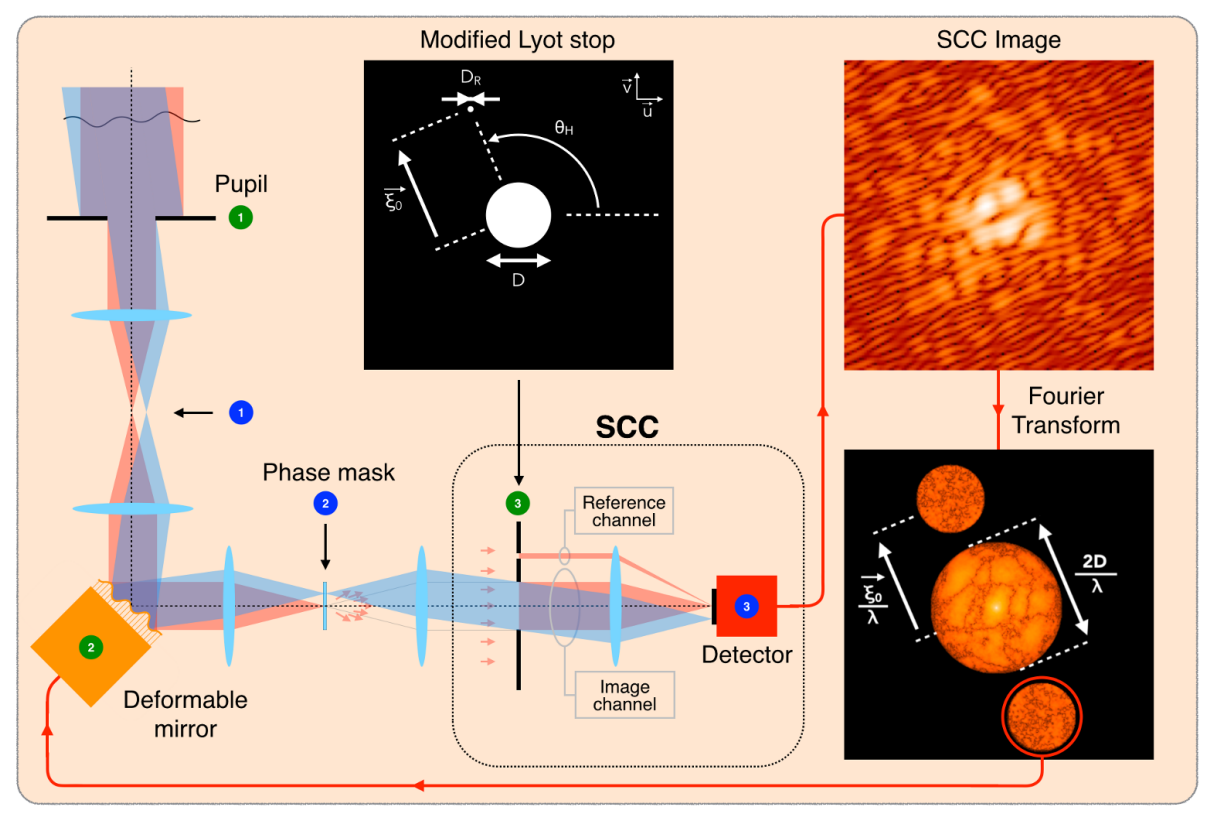

Fig. 1. Principle of the self-coherent camera (SCC). The stellar beam (red) hits a deformable mirror, and goes through a focal plane coronagraphic mask and a Lyot stop. Because of wavefront aberrations, part of the starlight goes through the image channel inducing speckles on the detector. Selecting part of the starlight rejected by the focal mask (reference channel), one spatially modulates the speckle intensity in the SCC image (top right). It is then possible to measure the speckle electric field from the Fourier transform of the SCC image (bottom right) in order to control the deformable mirror. The planet light (blue) is not affected by the coronagraph and the planet image is not fringed. system compensates for most of the atmospheric turbulence, but it cannot provide an aberration-free wavefront to the coronagraph. Moreover, the AO estimates aberrations in the wavefront sensing channel that is different from the science channel. Thus, NCPAs are seen by the coronagraph, which induce stellar speckles that mimic an exoplanet image in the science image. In space, optical aberrations vary because of variations in thermal or gravitational flexures. Hence, aberrations need to be calibrated regularly during the observations to have the coronagraph work in optimal conditions. To avoid NCPA or varying aberrations, focal plane wavefront sensors, which are the only efficient techniques, estimate the aberrations from the science image. Doing so means measuring the electric field associated with the stellar speckle in that plane. To this end, Bottom et al. (2017) implemented a phase-shifting interferometer on the stellar double coronagraph (SDC) to spatially modulate the speckle intensity. In this paper, we present the results obtained when implementing a self-coherent camera (SCC).

The SCC is a focal plane wavefront sensor that spatially modulates the intensity of the stellar speckles to retrieve the associated complex electric field (Baudoz et al. 2006, 2010, 2012; Galicher et al. 2008, 2010). It was optimized in the laboratory under spacelike conditions (Mazoyer et al. 2013, 2014; Galicher et al. 2014; Baudoz et al. 2018a) and ground-based conditions (Singh et al., in prep.). The principle is recalled in Fig. 1. The stellar beam (red) hits a deformable mirror. Then it is focused onto a coronagraphic focal plane mask that scatters light in the Lyot stop plane outside the geometrical pupil. A Lyot diaphragm stops the stellar light before it reaches the detector. If optical aberrations exist, part of the starlight is scattered inside the Lyot diaphragm and reaches the detector forming speckles that mimic an exoplanet image. The SCC adds a small hole in the Lyot stop (top image) to create a reference beam that interferes with the image channel and spatially modulates the stellar speckles in the science image recorded by the detector (top right). The SCC then does classical off-axis holography, and the lateral peak in the Fourier transform of that SCC image (bottom right) provides a direct estimation of the electric field in the science image. An interaction matrix is then recorded, and it is possible to control a deformable mirror to minimize the speckle intensity and to enhance the contrast in the science image (Mazoyer et al. 2013, 2014). If an exoplanet (blue beam) orbits the targeted star so that its image is not centered onto the focal plane mask, none of its light goes through the reference channel and its image is not fringed.

\section{Implementation in the stellar double coronagraph instrument}

The SDC instrument (Fig. 2, Mawet et al. 2014; Bottom et al. 2016 b) is installed at the primary focus of the Hale 200-inch telescope at Palomar. It is fed by the PALM-3000 adaptive optics system (Bouchez et al. 2008). It was designed to cancel the stellar light using two vortex coronagraphs in cascade (Mawet et al. 2011). After entering the SDC bench, the beam goes through Focal plane 1, Pupil plane 1, Focal plane 2, Pupil plane 2, and then it is injected in the PHARO system (Hayward et al. 2001).

The SCC has already been associated with numerous phase mask coronagraphs (Baudoz et al. 2018b) reaching very high contrast levels down to $4 \times 10^{-9}$ in space-like conditions (Baudoz et al. 2018a). In addition, as described in Sect. 2, implementing the SCC is as simple as adding a hole in the Lyot stop. This hole diameter is $\gamma$ times smaller than the science beam diameter $D$ and it is set at more than $1.5 \mathrm{D}$ from the center of the science beam. Hence, the optics after the Lyot stop must be twice the science beam so that both the science and the reference channels can propagate. This constraint is not a problem when designing a new instrument. However, it forbids the implementation of the SCC in most of the existing coronagraphic instruments because the optics after the Lyot stop are usually slightly larger (a few percentage points) than the science beam only.

To overcome this limitation and implement the SCC in the SDC instrument, we put no optics in focal plane 1 (Fig. 2). Doing so, the Hale pupil is reimaged in pupil plane 1 (Fig. 4). There, we add a diaphragm (represented by the blue circle) to create a $1.5 \mathrm{~m}$ off-axis pupil from the full $5 \mathrm{~m}$ obscured pupil. Then, we use the vortex phase mask of charge 2 in focal plane 2 . In pupil plane 2, we set up a reflective modified Lyot stop. The onaxis diaphragm stops the stellar light that is scattered by the vortex mask outside the geometrical pupil (classical coronagraphic Lyot stop in Fig. 3). The Lyot stop diameter is $88 \%$ of the offaxis pupil diameter to remove the light scattered near the border of the geometrical pupil. The SCC reference hole encircled 


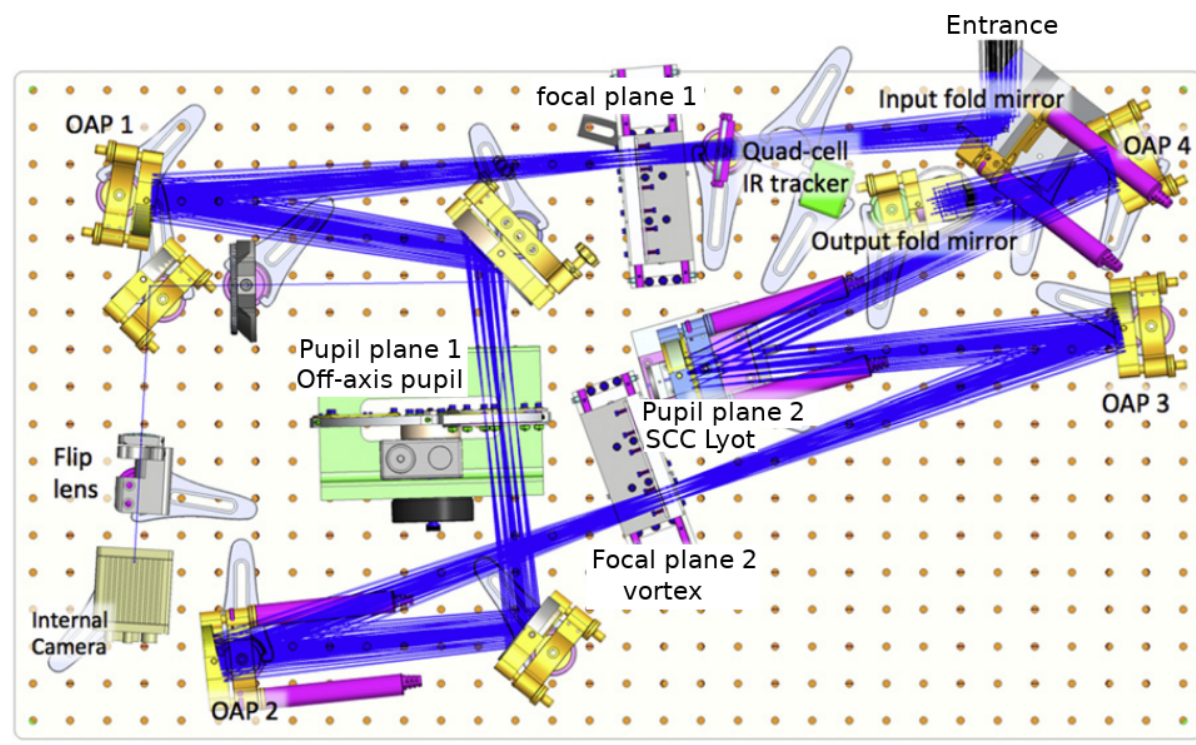

Fig. 2. Stellar Double Coronagraph (SDC) instrument layout. In Pupil plane 1, an off-axis pupil is set to simulate an off-axis $1.5 \mathrm{~m}$ telescope. A vortex phase mask is set in Focal plane 2 and a modified Lyot stop is installed in Pupil plane 2. The beam is then sent towards the Pharo detector.

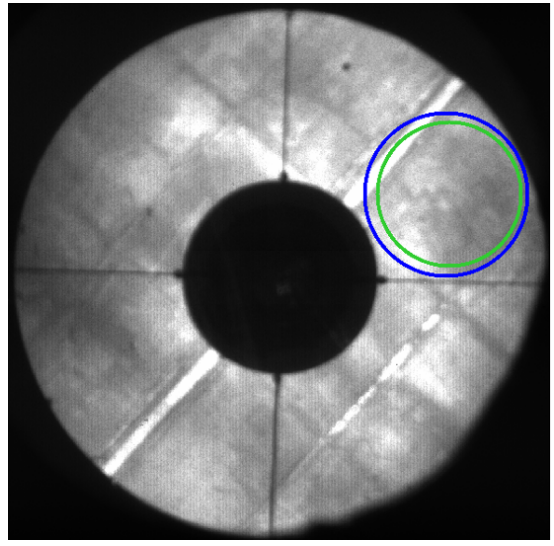

Fig. 3. Measured intensity in the Lyot stop plane when the beam of the internal source is centered on the vortex coronagraph. The classical Lyot stop is encircled in green. The SCC reference hole used during the run is encircled in red. The rest of the image should be dark as the Lyot mask should stop the star light. However, the mask is not perfectly black and reflected light is detected close to the Lyot stop.

in red in the figure has a diameter $\gamma=4$ times smaller than the Lyot stop. As explained in Mazoyer et al. (2013), the light of the reference beam mainly spreads in an Airy pattern with a radius of $\sim 1.2 \gamma \lambda / D$ in the coronagraphic image. Speckles are thus fringed up to $\sim 1.2 \gamma \lambda / D$ from the optical axis. Therefore, the smaller the value of $\gamma$, the larger the field of view that can be corrected from speckles. However, the larger the $\gamma$, the fainter the intensity of the reference beam in the coronagraphic image and the fainter the fringe visibility. A trade-off has to be made between the fringe visibility and the size of the corrected field of view. In the case of the SDC, there were large aberrations meaning there were bright speckles during our run. We had to use $\gamma=4$ so that the speckles close to the optical axis were correctly fringed. We could then minimize the speckle intensity within $5 \lambda / D$ of the optical axis. In order to enlarge the region of correction, we should have reduced the size of the reference hole (increasing $\gamma$ ), but we did not due to lack of time.

In Fig. 3, all but the Lyot stop and the reference disks should be dark as the Lyot mask should stop the star light. However, the mask is not perfectly black and reflected light is detected close to the Lyot stop.

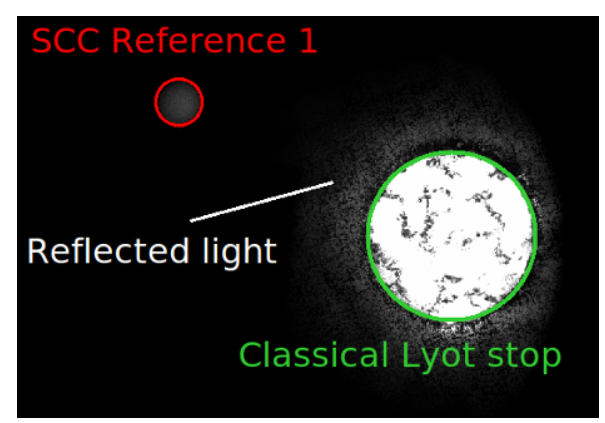

Fig. 4. Measured intensity in pupil plane when observing the dome of the Palomar observatory through the $5 \mathrm{~m}$ Hale telescope+SDC+Pharo. The blue circle represents the $1.5 \mathrm{~m}$ off-axis pupil that is selected in the first pupil plane in SDC. The green circle shows the position of the Lyot stop. The intensity pattern is the dome structure. The thin black horizontal and vertical lines are the spiders.

\section{NCPA correction procedure}

\subsection{MGS algorithm limitation}

At the Hale telescope, the current procedure used to minimize NCPA before the observations is based on a modified Gertzberg-Saxton (MGS) algorithm (Burruss et al. 2010). This technique estimates and minimizes the phase aberrations recording a set of out-of-focus non-coronagraphic images. The estimated aberrations include aberrations upstream and downstream of the coronagraphic focal plane mask, and they are both compensated by a deformable mirror that is upstream of the mask. The aberrations are thus overcorrected in the focal plane where the coronagraphic mask is. In addition, after an MGS minimization of NCPA, part of the stellar light leaks through the coronagraph and induces stellar speckles in the science image (see Sect. 5).

\subsection{SCC procedure}

To optimize the minimization of the speckle intensity in the science images, we used the SCC implemented in the SDC (Sect. 3) in closed-loop controlling the $66 \times 66$ Xinetics deformable mirror of the PALM-3000. As the SCC reference hole that we used is $\gamma=4$ times smaller than the Lyot diaphragm, the visibility of the SCC fringes was detectable up to $\sim 5 \lambda / D$ from the star 


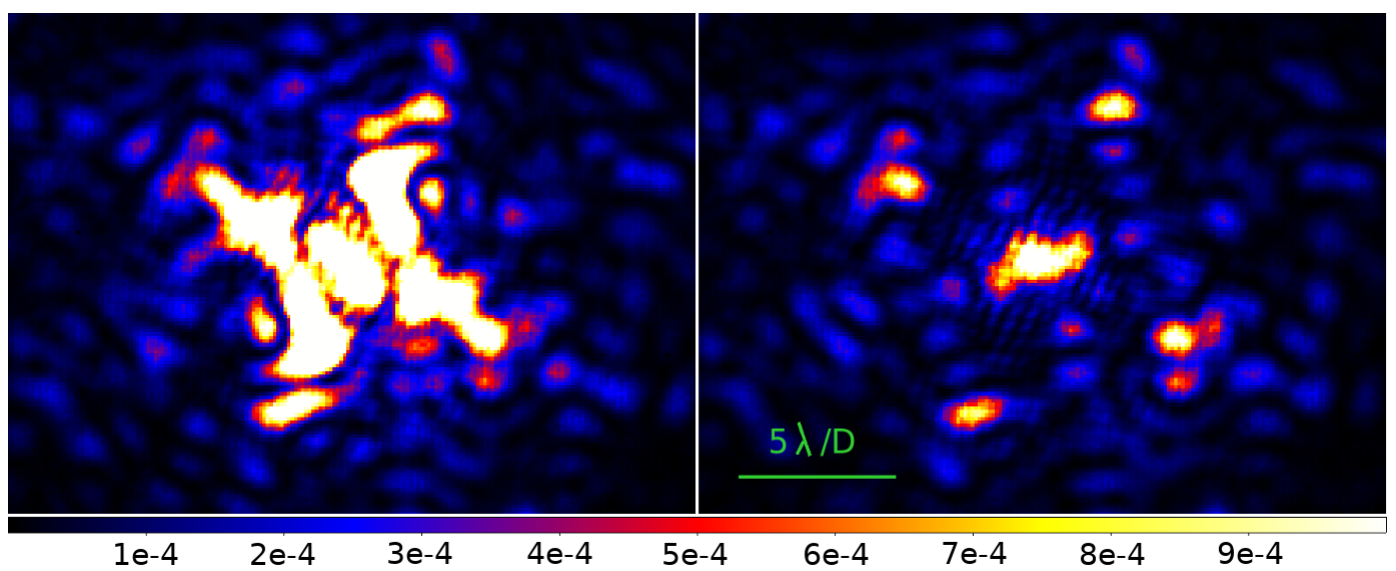

Fig. 5. Internal source: science images recorded at $\mathrm{Br}-\gamma$ after minimization of NCPA using the MGS algorithm (left panel) and using the SCC (right panel). The color scale and field of view are the same for both images. The color bar gives the intensity normalized by the non-coronagraphic PSF maximum.

in the science image (Mazoyer et al. 2013). Hence, we tried to minimize the speckle intensity up to $5 \lambda / D$. To correct at larger separations, we would need to use a smaller reference hole so that the reference intensity nulls farther away.

One iteration of correction consists on recording one SCC coronagraphic science image, estimating the electric field associated with the stellar speckles from this image (Sect. 2), and sending commands to the deformable mirror using the control matrix. The control matrix is the pseudo-inverse of the interaction matrix that we recorded using the internal source of the SDC instrument. To record this matrix, we used a truncated Fourier basis (Mazoyer et al. 2014) composed of all sine and cosine functions in the pupil plane that induce speckles below $5 \lambda / D$ in the science image. One row of the interaction matrix is the estimated electric field in the science image when applying one function of our basis. To apply the sine and cosine phase functions, we modified the voltages of the deformable mirror. Recording this matrix with the Pharo detector took about $20 \mathrm{~min}$. Once this calibration was done, we could close the SCC correction loop to minimize the NCPA. We note that we assume small aberrations when recording the interaction matrix. As a consequence several iterations of correction are needed to minimize the speckle intensity.

\section{Internal source}

\subsection{Performance}

We were granted two nights at the Hale telescope on the Stellar Double Coronagraph (program 3660 ${ }^{1}$ ). During daytime on 25 July 2018, we first minimized the NCPA in the SDC instrument using the MGS algorithm using the Br- $\gamma$ filter $\left(\lambda_{0}=2.166 \mu \mathrm{m}\right.$ and $\Delta \lambda=0.020 \mu \mathrm{m}$, Hayward et al. 2001) and the internal source. NCPA were reduced, but speckles were still present in the MGS coronagraphic image (left panel in Fig. 5) because the MGS solution overcorrects the aberrations upstream of the coronagraph focal plane mask, as explained in Sect. 4.1.

We then used the SCC to optimize the speckle minimization. We started from the MGS image (left panel in Fig. 5) in which the SCC fringes are detected (from bottom left to top right). We estimated and corrected the aberrations up to $5 \lambda / D$ around the optical axis. After three iterations the speckle intensity inside the

\footnotetext{
1 https://reservations.palomar.caltech.edu/observing_ schedule/abstract/3660
}

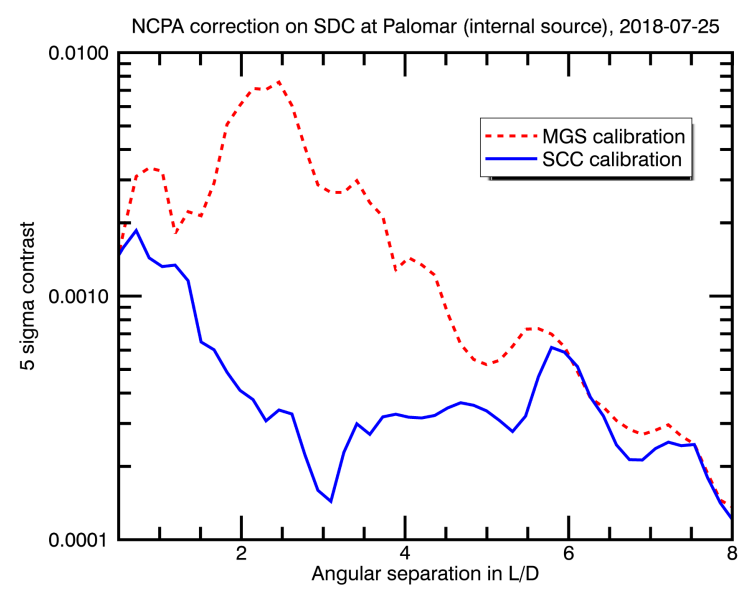

Fig. 6. Internal source: $5 \sigma$ detection limit associated with MGS (red dashed line) and SCC (blue full line) calibrated images of Fig. 5.

control area was efficiently reduced, as shown in the right panel in Fig. 5. The four satellite speckles are also present in the MGS image. They are between $\sim 5 \lambda / D$ and $\sim 6 \lambda / D$, and then outside the corrected area, which is why they are not corrected in the SCC image.

The $5 \sigma$ detection limit for the MGS and the SCC calibrated images are plotted in Fig. 6 . The detection limit is the azimuthal standard deviation of the intensity calculated in annuli of $1 \lambda / D$ width and centered on the optical axis (i.e., the star image). The self-coherent camera improves the detection limit in the coronagraphic image by a factor of $4-20$ between 1.5 and $5 \lambda / D$. This result demonstrates the efficiency of the SCC very close to the optical axis where many exoplanets have yet to be discovered and where other techniques like angular and spectral differential imaging cannot calibrate the speckles (Marois et al. 2006; Racine et al. 1999).

\subsection{Comparison with other techniques}

Other techniques like reference differential imaging (Ruane et al. 2019) can partially calibrate the speckles close to the optical axis. They are however limited by speckles with lifetimes shorter than a few minutes; the speckle pattern then changes between the science target and the reference star. Other techniques can do focal plane wavefront sensing and correction (Martinache et al. 2014; 


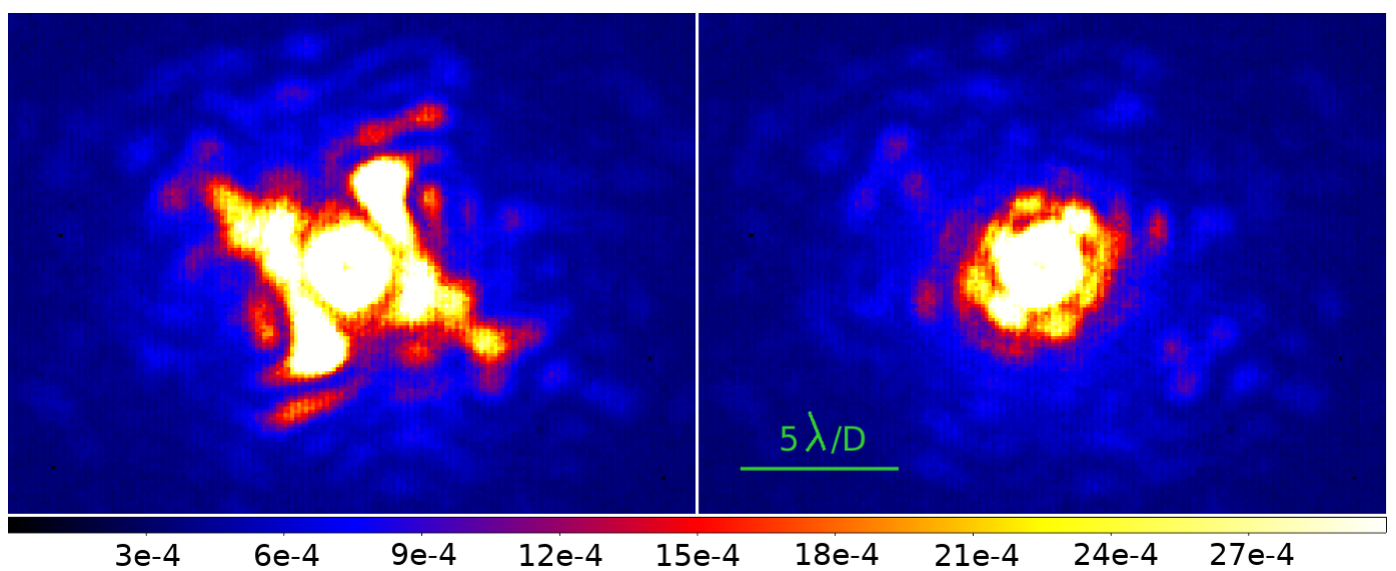

Fig. 7. On-sky: science SCC coronagraphic images at Br- $\gamma$ using the MGS solution (left panel) or the SCC solution (right panel). The color scale and field of view are the same for both images. The color bar gives the intensity normalized by the non-coronagraphic PSF maximum.

Bottom et al. 2016a, 2017; Cady et al. 2013; Matthews et al. 2017). Most of them use a temporal modulation of the speckle intensity and they cannot calibrate the speckles with lifetimes shorter than the time needed for calibration, usually 4 to 5 images, meaning a few minutes for a typical star magnitude.

\subsection{Current limitation at Palomar}

The contrast level reached in the SDC images (a few $10^{-4}$ ) is moderate when compared to results obtained in the laboratory (down to $4 \times 10^{-9}$, Baudoz et al. 2018a). Close to the optical axis, the performance is set by the SDC vortex phase mask, the reflected light by the Lyot stop (see the end of Sect. 3), and the jitter stability during the exposure that is at least $\sim 1.5 \mathrm{~s}$ (plus $\sim 6.5 \mathrm{~s}$ of overhead). We note, however, that the contrast we obtained in Sect. $5.1\left(\sim 2 \times 10^{-3}\right)$ is better than that previously reached using the same $1.5 \mathrm{~m}$ off-axis configuration $\left(5 \times 10^{-3}\right.$ to $10^{-2}$ in Serabyn et al. 2010).

Farther away from the optical axis, we believe that the performance can be improved in the current SDC instrument reducing the reflected light by the Lyot stop and optimizing the SCC speckle calibration, but we had a limited amount of time at the telescope. However, improving the performance in SDC images does not mean reaching $4 \times 10^{-9}$ contrast levels. This level of performance is possible using a coronagraph that reaches a contrast of a few $10^{-5}$ on the optical axis in a system that remains stable during the speckle calibration (gravity and thermal flexures inducing a jitter smaller than $\sim \lambda /(10 D)$ ). This would be possible using a faster detector than Pharo, whose highest rate is about $0.1 \mathrm{~Hz}$.

\section{On-sky performance}

During our stay at the Palomar Observatory, the quad-cell infrared tracker that is used to stabilize the star image at the center of the vortex phase mask (i.e., control of the jitter, Bottom et al. 2016b) was not in service. Therefore, it was not possible to close the SCC loop on-sky. Moreover, even if the quad-cell tracker was used, a faster detector than Pharo would be a key point to control the speckles before they evolve because of gravity or thermal flexures.

As we could not close the loop on-sky, we used another approach. On 25 July 2018 we minimized the speckle intensity on internal source during daytime using the MGS algorithm and the SCC technique. Between $3 \mathrm{~h}$ and $4 \mathrm{~h}$ later we opened the

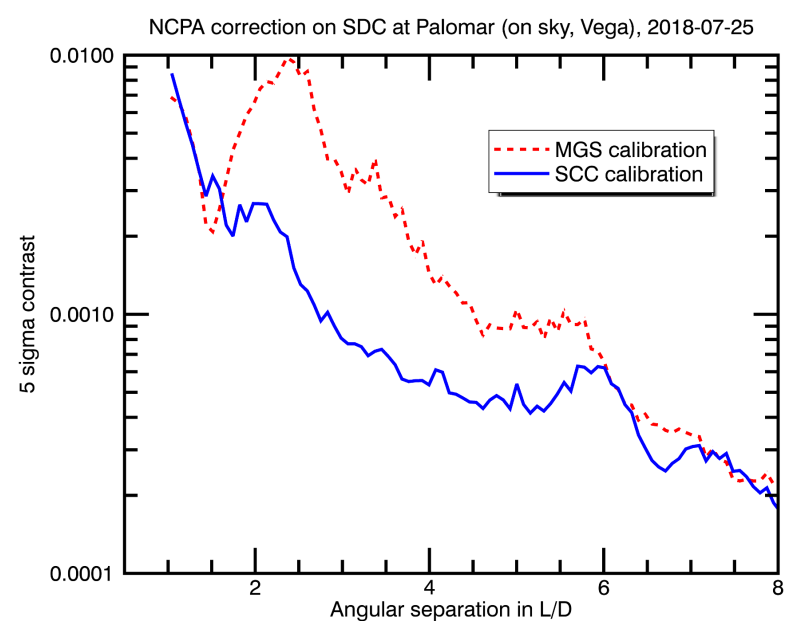

Fig. 8. On-sky: $5 \sigma$ detection limit associated with MGS (red dashed line) and SCC (blue solid line) calibrated images from Fig. 5.

telescope and pointed at Vega with a seeing of $\sim 1.7$ arcsec. We recorded sequences of images in $\operatorname{Br} \gamma$ with the beam aligned on the vortex coronagraph.

During the first sequence, we applied the MGS calibration described in Sect. 5. We recorded 40 exposures of $1.416 \mathrm{~s}$. The average of the coronagraphic images is shown in the left panel in Fig. 7. The image is very similar to the one measured with the internal source, except close to the center. This difference is due to uncorrected jitter when on-sky.

During the second sequence we applied the SCC solution obtained with the internal source and we recorded 20 exposures of $1.416 \mathrm{~s}$. The averaged image is shown in the right panel in Fig. 7. As with the internal source, the quality of the image is clearly improved with respect to the MGS solution. Speckles are suppressed from the image. This is confirmed when plotting the $5 \sigma$ detection limit (Fig. 8). Between 2 and $4 \lambda / D$, the detection limit is $\sim 5$ times better after SCC calibration than after MGS calibration. The differences with the image obtained on an internal source (Sect. 5) are the starlight leakage close the star center due to uncorrected jitter, a smooth halo created by averaging turbulence speckles, and residual speckles above this halo up to $\sim 5 \lambda / D$. The last can be induced by uncorrected static aberrations due to telescope aberrations that were not calibrated using the internal source. 


\section{Conclusion}

To improve exoplanet imaging instruments, a crucial point is to actively compensate for the non-common path aberrations (NCPA) between the classical adaptive system channel and the science coronagraphic image. The use of a focal plane wavefront sensor is the unique technique for estimating the electric field of the stellar speckles from the coronagraphic image. Such a sensor is the self-coherent camera (SCC) that spatially modulates the speckle intensity. To implement the SCC on the existing stellar double coronagraph at the 200" Palomar telescope, we added a small off-axis hole in the Lyot stop of the vortex coronagraph.

Using the SCC, we improved the detection limit between 1.5 and $5 \lambda / D$ by a factor of 4 to 20 in the laboratory using the internal source. We then tested on-sky the quality of the internal calibration. We observed Vega and showed that the SCC calibration was five times better between 2 and $4 \lambda / D$ when compared to the Palomar standard calibration used for NCPA minimization. The loss of performance of the SCC calibration between on-sky and on internal source may come from residual unaveraged aberrations in long but not infinite exposures. Additional telescope time is needed to investigate this issue.

To conclude, we demonstrated the capacity of the selfcoherent camera to calibrate NCPA in an existing instrument. This first demonstration was made using a narrowband filter, but implementation of the SCC in broadband is possible using several SCC reference holes, as explained in Delorme et al. (2016). The results related in this paper also highlight two interests of the SCC. First, the SCC can minimize the speckle intensity in the field of view, especially when very close to the star where many exoplanets have yet to be discovered. Then, even if the SCC requires a 3-4 pixel sampling on the science detector instead of 2 for other focal plane wavefront sensors, the SCC has a $100 \%$ efficiency with science time as each image can be used for both science and NCPA minimization.

Acknowledgements. The authors thank the Région Île-de-France and the Science Council of the Paris Observatory for their support of this work.

\section{References}

Baudoz, P., Boccaletti, A., Baudrand, J., \& Rouan, D. 2006, in Direct Imaging of Exoplanets: Science Techniques, eds. C. Aime, \& F. Vakili, IAU Colloq. 200, 553

Baudoz, P., Mas, M., Galicher, R., \& Rousset, G. 2010, SPIE Conf. Ser., 7736, $77365 \mathrm{X}$

Baudoz, P., Mazoyer, J., Mas, M., Galicher, R., \& Rousset, G. 2012, SPIE Conf. Ser., 8446,84468
Baudoz, P., Galicher, R., Potier, A., et al. 2018a, in Advances in Optical and Mechanical Technologies for Telescopes and Instrumentation III, SPIE Conf. Ser., 10706, 1070620

Baudoz, P., Galicher, R., Patru, F., Dupuis, O., \& Thijs, S. 2018b, ArXiv e-prints [arXiv: 1801.06600 ]

Beuzit, J. L., Vigan, A., Mouillet, D., et al. 2019, A\&A, accepted, https:// doi.org/10.1051/0004-6361/201935251

Bordé, P. J., \& Traub, W. A. 2006, ApJ, 638, 488

Bottom, M., Femenia, B., Huby, E., et al. 2016a, in Adaptive Optics Systems V, Proc. SPIE, 9909, 990955

Bottom, M., Shelton, J. C., Wallace, J. K., et al. 2016b, PASP, 128, 075003

Bottom, M., Wallace, J. K., Bartos, R. D., Shelton, J. C., \& Serabyn, E. 2017, MNRAS, 464, 2937

Bouchez, A. H., Dekany, R. G., Angione, J. R., et al. 2008, in Adaptive Optics Systems, SPIE Conf. Ser., 7015, $70150 Z$

Burruss, R. S., Serabyn, E., Mawet, D. P., et al. 2010, in Adaptive Optics Systems II, Proc. SPIE, 7736, 77365X

Cady, E., Baranec, C., Beichman, C., et al. 2013, in Techniques and Instrumentation for Detection of Exoplanets VI, Proc. SPIE, 8864, 88640K

Delorme, J. R., Galicher, R., Baudoz, P., et al. 2016, A\&A, 588, A136

Galicher, R., Baudoz, P., \& Rousset, G. 2008, A\&A, 488, L9

Galicher, R., Baudoz, P., Rousset, G., Totems, J., \& Mas, M. 2010, A\&A, 509, A31

Galicher, R., Baudoz, P., Delorme, J. R., et al. 2014, in Space Telescopes and Instrumentation 2014: Optical, Infrared, and Millimeter Wave, SPIE Conf. Ser., 9143, 91435A

Give'on, A., Kern, B., Shaklan, S., Moody, D. C., \& Pueyo, L. 2007, in Astronomical Adaptive Optics Systems and Applications III, Proc. SPIE, 6691, 66910A

Give'on, A., Kern, B. D., \& Shaklan, S. 2011, in Techniques and Instrumentation for Detection of Exoplanets V, Proc. SPIE, 8151, 815110

Hayward, T. L., Brandl, B., Pirger, B., et al. 2001, PASP, 113, 105

Lawson, P. R., Belikov, R., Cash, W., et al. 2013, in Techniques and Instrumentation for Detection of Exoplanets VI, Proc. SPIE, 8864, 88641F

Macintosh, B., Graham, J. R., Ingraham, P., et al. 2014, Proc. Natl. Acad. Sci., 111,12661

Marois, C., Lafrenière, D., Doyon, R., Macintosh, B., \& Nadeau, D. 2006, ApJ, 641, 556

Martinache, F., Guyon, O., Jovanovic, N., et al. 2014, in Adaptive Optics Systems IV, Proc. SPIE, 9148, 914821

Matthews, C. T., Crepp, J. R., Vasisht, G., \& Cady, E. 2017, J. Astron. Telesc. Instrum. Syst., 3, 045001

Mawet, D., Serabyn, E., Wallace, J. K., \& Pueyo, L. 2011, Opt. Lett., 36, 1506

Mawet, D., Shelton, C., Wallace, J., et al. 2014, in Space Telescopes and Instrumentation 2014: Optical, Infrared, and Millimeter Wave, Proc. SPIE, 9143, 91432T

Mazoyer, J., Baudoz, P., Galicher, R., Mas, M., \& Rousset, G. 2013, A\&A, 557, A9

Mazoyer, J., Baudoz, P., Galicher, R., \& Rousset, G. 2014, A\&A, 564, L1

Racine, R., Walker, G. A. H., Nadeau, D., Doyon, R., \& Marois, C. 1999, PASP, 111,587

Ruane, G., Ngo, H., Mawet, D., et al. 2019, AJ, 157, 118

Serabyn, E., Mawet, D., \& Burruss, R. 2010, Nature, 464, 1018

Snik, F., Absil, O., Baudoz, P., et al. 2018, in Advances in Optical and Mechanical Technologies for Telescopes and Instrumentation III, SPIE Conf. Ser., 10706, 107062L 